\title{
Performance Improvement of MIMO-OSTBC System with BCH-TURBO Code In Rayleigh Fading Channel
}

\author{
Sofi Naima ${ }^{1}$, Debbat Fatima ${ }^{2}$, Bendimerad Fethi. Tarik ${ }^{3}$ \\ ${ }^{1,3}$ Department of telecommunications, LTT laboratory, university of tlemcen, Algeria \\ ${ }^{2}$ Department of informatics, Mascara University, Algeria
}

\begin{tabular}{l}
\hline \hline Article Info \\
\hline Article history: \\
Received Oct 9, 2017 \\
Revised May 15, 2018 \\
Accepted Jun 7, 2018 \\
\hline Keywords: \\
BCH \\
BCH-TC \\
CC \\
MIMO \\
OSTBC \\
RS \\
TC \\
\hline
\end{tabular}

\begin{abstract}
Recently, OSTBCs has become a widespread technique for signal transmission over wireless channels because of their diversity gain, but there are not designed to achieve an additional coding gain. Hence, OSTBCs must be concatenated with an external code which allows a significant coding gain. FEC (forward error correction) is a technique used for detecting and possibly correcting errors that can occur when messages are transmitted through a digital communication system, also for rendering the information more reliable. Thus, with staffing these coding techniques that are able to reach Shannon limits, in MIMO systems, better performances can be achieved by taking advantages of diversity and coding gains. The objective of this paper is to compare different FEC codes in Rayleigh fading channel and propose an appropriate code for MIMO-OSTBC systems. The simulation results reveal the performance of the proposed model.
\end{abstract}

Copyright (C) 2018 Institute of Advanced Engineering and Science. All rights reserved.

\section{Corresponding Author:}

Sofi Naima,

Department of telecommunications,

LTT laboratory, university of tlemcen, Algeria.

Email: dr_sofi_naima@hotmail.com

\section{INTRODUCTION}

In recent decades, and because of the ever increasing demand for efficient and reliable digital transmission, new methods have emerged to meet this massive commun ications demand under constraints of limited bandwidth and transmit power.

MIMO technology is one of the most important research areas in communications, it uses multiple antennas in transmitter and/or receiver, and it can achieve the high diversity gain through STC and high data rate with spatial multiplexing [1] and also assures high speed transmission with a minimum loss in quality of services. Intense research activity has appeared on MIMO systems and it was inspired by different works of Telatar and Foschini [2].

Space time block codes (STBCs) from orthogonal design are attractive since they achieve the maximum diversity gain and the highest throughput it is an elegant method for transmission using multiple transmit antennas. And these codes have a simple decoding algorithm which is only based on linear processing [3], [4].

Despite its advantages, this coding system does not generally provide coding gain. Unless concatenated with an outer code.

In this context, several studies have been done on the concatenation of FEC and STBC that try to find a good combination with minimum complexity and with high coding gain as with high error correction. In [5] different schemes of concatenated STBC and channel coding as TCM (trellis codes modulation), CC (Convolutional Code), turbo, turbo $\mathrm{BCH}$ and Turbo TCM was been analyzed and it showed that TC (turbo 
codes) gave the best coding gain with a moderate complexity. In [6] concatenation of STBC and LDPC (low density parity check) was studied and it was shown that this concatenation provide a coding gain of $9 \mathrm{~dB}$ at $10^{-4}$ as compared with uncoded STBC and in [7] new concatenated scheme was developed to perform better performance. As well as in [8], where a novel hybrid space time blocks coding scheme was studied and compared with an uncoded STBC and it proves better performance.

In this paper, we study different FEC concatenated with OSTBC to show the efficient combination. Because of turbo codes performance which approaches the Shannon limit and its stability for long time [9]. And as it is shown in [10] about the efficiency of an outer BCH code in the improvement of turbo code performance, we propose a new concatenation BCH-TURBO code with OSTBC systems where BCH and TURBO are serially concatenated without interleavers between them and then concatenated with OSTBC to improve their capacity and to obtain an additional coding gain. By dint of its constant complexity and its performance in error correction, this scheme can be employed in multitude of applications such as those related to satellites as well as those relating to deep space communications, wireless networks and also mobile communications.

This paper is organized as fellows, in Section II a brief introduction to OSTBC with explanation of encoder and decoder, section III: introduction to some necessary concepts about different coding techniques. Section IV an overview of our model turbo-BCH-OSTBC, the simulation results of different FEC in concatenation with OSTBC in comparison with our proposed model and conclusion are detailed in Section V and VI respectively.

\section{THE COMPREHENSIVE THEORETICAL BASIS}

\subsection{OSTBC System}

In wireless multipath environment, severe attenuations prevent the receiver to determine the transmitted signal, unless some less attenuated replicas of the transmitted signal is provided to receiver when the receiver is equipped with some form of diversity [4].

However, at the station, we must combine the received symbols of the different propagation paths. Which implies more complexity, in a way to motivates and consider transmit diversity with an optimal scheme appealing in term of simplicity and performance discovered by Alamouti [3], called STB codes and it motivates search for similar scheme using more than 2 transmit antennas.

Tarokh, Jafarkhani and Calderbank apply the theory of orthogonal design to generalize Alamouti's STBC for more than 2 antennas and create OSTBC with a very simple Maximum-Likely-hood decoding and that have full diversity $\left(\mathrm{N}_{\mathrm{t}} \times \mathrm{N}_{\mathrm{r}}\right)$ [11].

\subsubsection{OSTBC Encoding Algorithm}

We consider a wireless communication system with $\mathrm{N}$ antennas in transmitter and $\mathrm{M}$ antennas in receiver sides. In a space time block coding scheme, the signal are encoded in a transmission matrix $G$ with order $\mathrm{x}$ N [11].

Where the entries of the matrix are linear combinations of variables $x_{1}, x_{2}, \ldots, x_{k}$, and their conjugates.

$$
G_{2}=\left(\begin{array}{cc}
x_{1} & x_{2} \\
-x_{2}^{*} & x_{1}^{*}
\end{array}\right)
$$

The space time block code $G_{2}$ uses the transmission matrix in (1). Let us consider that we have $2^{b}$ signals in the constellation. $2^{b}$ bits arrive at the encoder and select two complex symbols $s_{1}, s_{2}$ and they are transmitted from the first and the second antenna respectively, at the first time slot [11].

\subsubsection{OSTBC Decoding Algorithm}

At time $t$ the signal $r_{t}^{j}$, received at antenna $j$, is given by

$$
r_{t}^{j}=\sum_{i=1}^{n} \alpha_{i, j} C_{t}^{j}+\eta_{t}^{j}
$$

The $\eta_{t}^{j}$ are independent samples of a zero-mean complex Gaussian random variable with variance $n /(2 S N R)$ per complex dimension. And $C_{t}^{j}$ are coded signals. $s_{1}$ and $s_{2}$

The maximum likelihood detector minimizes the decision metric over all possible values of signals

$$
\sum_{j=1}^{m}\left(\left|r_{1}^{j}-\alpha_{1, j} s_{1}-\alpha_{2, j} s_{2}\right|^{2}+\left|r_{2}^{j}+\alpha_{1, j} s_{2}^{*}-\alpha_{2, j} s_{1}^{*}\right|^{2}\right)
$$


After minimization of $s_{1}$ and $s_{2}$, the resulted values are the estimates of them.

After expanding and deleting the independent terms of the code words of the Equation (3) , and decomposition of the result equation in two parts one of them is a function of $s_{1}$ and the other is function of $s_{2}$. [3] [11].

The detection of $\mathrm{s} 1$ amounts to minimize the decision metric:.

$$
\left|\left[\sum_{j=1}^{m}\left(r_{1}^{j} \alpha_{1, j}^{*}+\left(r_{2}^{j}\right)^{*} \alpha_{2, j}\right)\right]-s_{1}\right|^{2}+\left(-1+\sum_{j=1}^{m} \sum_{i=1}^{2}\left|\alpha_{i, j}\right|^{2}\right)\left|s_{1}\right|^{2}
$$

And the minimization of :

$$
\left|\left[\sum_{j=1}^{m}\left(r_{1}^{j} \alpha_{2, j}^{*}-\left(r_{2}^{j}\right)^{*} \alpha_{1, j}\right)\right]-s_{2}\right|^{2}+\left(-1+\sum_{j=1}^{m} \sum_{i=1}^{2}\left|\alpha_{i, j}\right|^{2}\right)\left|s_{2}\right|^{2}
$$

for the decoding of $s_{2}$. This is the simple decoding scheme described in [11] , and there is no performance sacrifice for using it.

\subsection{Concatenated OSTBC with Channel Coding Techniques}

OSTBC provide important diversity advantages at a low decoding complexity. However, they were not designed to achieve additional coding gain, unless concatenated with an outer code.

On the other hand, powerful coding channel is essential to fight against the effects of fading, interference and noise to obtain sufficient quality of reception. The first idea of concatenated code was conceived by Forney [12] to find a code that has decrease error probability with increasing of block length the block diagram of this system is presented in Figure 1.

Convolutional coding and block coding are the two major forms of channel coding. Convolutional codes operate on serial data, which means one or a few bits at a time. The Block codes operate on relatively large message blocks. There are a variety of useful convolutional and block codes, and also a variety of algorithms for decoding the received coded information sequences to recover the original data. The investigated channel codes in this paper are $\mathrm{CC}, \mathrm{RS}, \mathrm{BCH}, \mathrm{TCM}$ and turbo code.

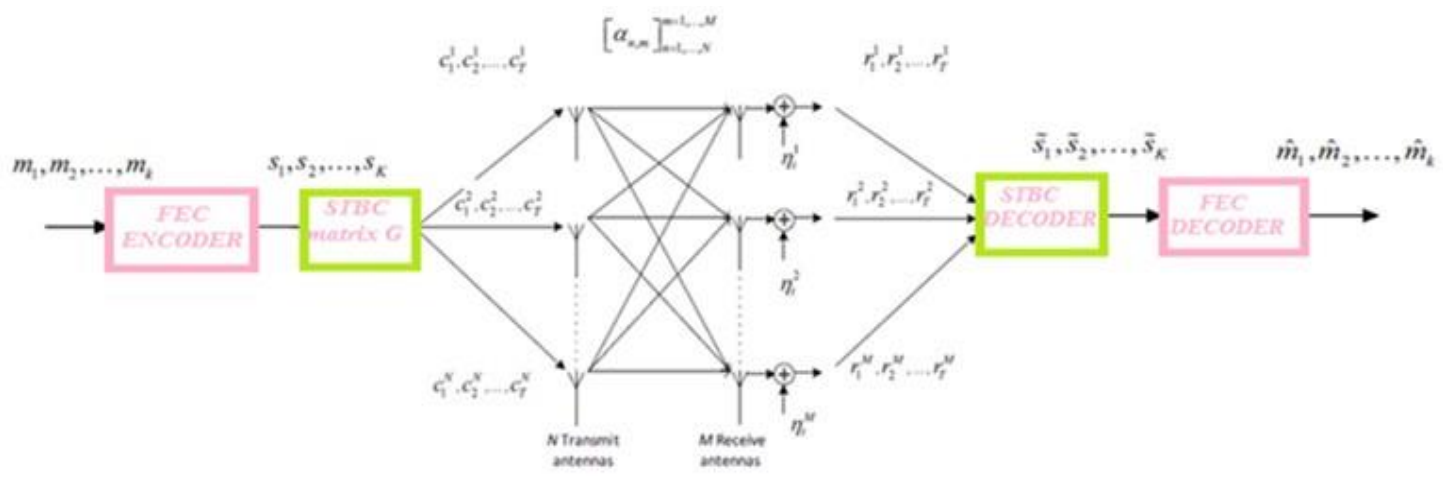

Figure 1. concatenated STBC with FEC in MIMO system

\subsubsection{Convolutional Code}

One of the first practical FEC codes was the convolutional codes that were discovered by Elias in 1955.it is a special case of error control coding, it is not memory less. In addition, the convolutional coder accepts a fixed number of message symbols and produces a fixed number of code symbols, its computations depend not only on the current set of input symbols but on some of the previous input symbols too.

Convolutional codes are commonly specified by three parameters (n,k,m) [14];

$\mathrm{n}=$ number of input bits

$\mathrm{k}=$ number of output bits

$\mathrm{m}=$ number of registers 
And can be specified by an additional parameter L (constraint length) $\mathrm{L}=\mathrm{k}(\mathrm{m}-1)$. The Figure 2 shows an example of convolutional encoder with one input, two outputs, and two shift registers.

Each input bit is coded into $m$ output bits, the $m$ output bits are produced by $m$ modulo-Ladders by adding up certain bits in the memory registers. The selection of which bits that are going to be added to produce the output bits is called "the generator polynomial (g)" for that output bit, and the output bites are just the sum of these bits. The polynomial gives the code its unique error protection quality.

The decoding algorithm for the convolutional code is called Viterbi algorithm, it was created by Viterbi in 1967 [15]. Another decoding algorithm was also created by Bahl and al. called MAP algorithm [16] that outperform the VA in BER terms.

In [17], [18] and [19] the concatenation of STBC with convolutional code was studied and as conclusion they prove that STBC combined with convolution code can improve the transmission efficiency with a modified Viterbi algorithm.

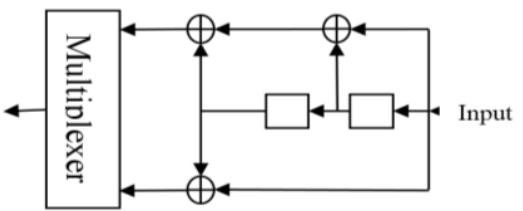

Figure 2. Convolutional Encoder [13]

\subsection{2. $\quad$ BCH}

Block coding is a special case of error-control coding. Block-coding techniques map a fixed number of message symbols to a fixed number of code symbols. A block coder treats lso each block of data independently and it is a memory less.

$\mathrm{BCH}$ codes were discovered independently by Bose and Ray-Chaudhuri [20] and by Hocquenghem [21] in the late 1950s.

$\mathrm{BCH}$ codes are characterized by the following parameters for any positive integer's $m$ where $m \geq 3$ and $t$ where $t<2^{m}-1$ there exist a binary $\mathrm{BCH}$ code where

Block Length: $\mathrm{n}=2^{\mathrm{m}}-1$

Number of Parity-Check digits: $r=n-k \leq m t$

Minimum distance: $\mathrm{dmin} \geq 2 \mathrm{t}+1$.

The Alphabet of a $\mathrm{BCH}$ code for $\mathrm{n}=2^{\mathrm{m}}-1$ is represented as the set of elements of an appropriate Galois field, GF (2m) where primitive element is $\alpha$ [22]. For decoding $\mathrm{BCH}$ encoded signals, $\mathrm{BCH}$ decoder uses Berlekamp Algorithm [23].The effect of $\mathrm{BCH}$ in some systems has been examined in several works. In [24], the implementation of BCH in STBC system is studied. It is also demonstrated in [25] that $\mathrm{BCH}$ Code improves the BER performance in a hybrid multiple access scheme.

\subsubsection{Reed Solomon}

The RS code can be considered as a non-binary $\mathrm{BCH}$ code, $\mathrm{RS}$ are $\mathrm{BCH}$ codes where values of the code coefficients are taken from Galois Field (GF) $\left(2^{\mathrm{m}}\right)$.

With the addition of $t$ check symbols to the data, detection of any combination of up -to t erroneous symbols and correction up to [t/2] symbols can be made by RS codes. These codes differentiate from a Hamming code in the fact that it encodes groups of bits rather than encoding one bit at a time [26].

On the same point, the RS codes are very efficient and best way to correct several errors in a short time and have a wide range of applications in digital communication.

An RS-code is specified as $R S(n, k, t)$ with $l$ bit symbols. This means that the encoder takes $k$ data symbols of $l$ bits each and adds $2 t$ parity symbols to construct an $n$-symbol codeword.

Therefore: $\mathrm{n}$ is the number of bytes after encoding; $k$ is the number of data bytes before encoding, and $t$ is the number of data bytes that can be corrected. Then RS-code can correct up to $t$ symbols, where $t$ can be expressed as $t=(n-k) / 2$.

After the RS encoding process, data bits are further encoded by the STBC encoder. It u ses Berlekamp-Massey algorithm for decoding.

In [27] the concatenation of RS code and STBC has been analyzed, and it gave a conclusion that this combination has an effect in improvement of channel reliability. In [28], also this combination was analyzed and compared with cc-STBC in WIMAX system. 


\subsubsection{TCM}

The TCM scheme proposed by Ungerboeck in his original paper in IEEE (1982). The functions of a TCM consist of a Trellis Code and a Constellation Mapper as shown below in Figure 3.

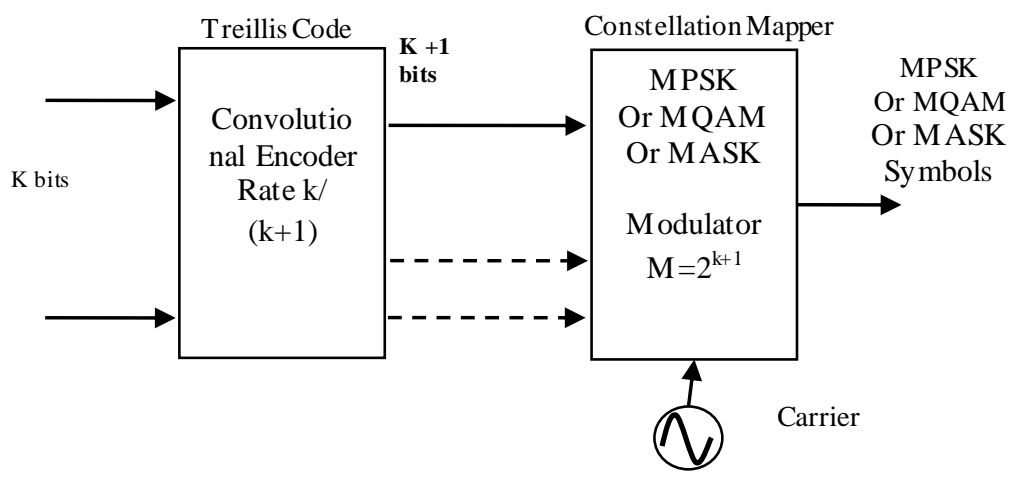

Figure 3. TCM Code [29]

TCM is a convolutional coding. Where not all incoming bits are coded and only 1 extra bit is always added. The increasing of the constellation size reduces Euclidean distances between the constellation points, but otherwise, sequence coding offers a coding gain that overcomes the power disadvantage of going to the higher constellation. The decoding metric is the Euclidean distance and not the Hamming distance. TCM uses set-partitioning and small number of states [29].

The TCM decoder uses soft-decision decoding to find the path with minimum (squared) Euclidean distance through the trellis. This makes the trellis code design trying to maximize the Euclidean distance among the code words, and the set partitioning let the most significant message bit(s) have a larger Euclidean distance from its complement so that there are less likely errors in the uncoded message bits than in the coded $\operatorname{bit}(s)$ [29].

Several concatenation have been done to improve the channel performance and to find suitable coding scheme for STBC in MIMO system as shown in [30], [31].

\subsubsection{Turbo Code}

The turbo code was invented by Berrou in 1993 [9], that code consists of two or more convolutional code connected in parallel with pseudo random interleavers.

As in Figure 4, the turbo code (with block length $\mathrm{K}$ ) is composed of two Recursive Systematic Convolutional codes, where the information bits are fed to the first RSC and after being interleaved are passed through the second constituent encoder. The resulting codeword consists of the systematic bits, $\mathrm{k}(\mathrm{i})$, and two parity check streams, $r 1(i), r 2(i), i=1,2, \ldots, K$.

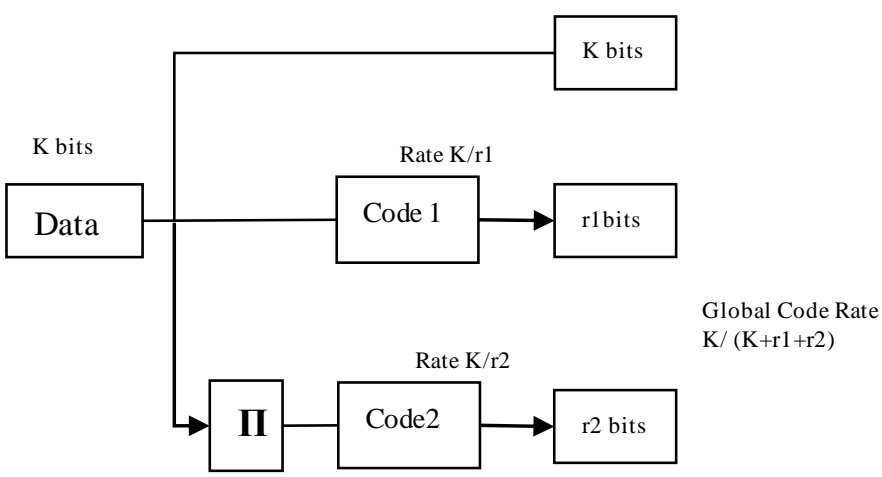

Figure 4. Basic Structure of the Turbo Encoder [30] 
For the turbo decoder as it is shown below in Figure 5, it consists of two concatenated decoders, each one provides a soft output of the transmitted bits by using the received data and the information provided through the other decoder, this soft output is APP [16].

The extrinsic information produced by the other constituent decoder is used to evaluate the APP at each iteration. The decoder use two efficient algorithm BCJR presented in [16] and the suboptimal decoder presented in [9].

Several concatenation of turbo code with STBC was studied and it proves that this scheme provide a good performance improvement in term of BER as shown in [33].

Figure 6 shows a simulation result of OSTBC turbo codes with various numbers of iterations, it can be observed that using turbo code only can produce a good performance. it needs only $10 \mathrm{db}$ of EbNo to achieve less than $10^{-5}$ BER with 6iterations and it can be decreased even much more by increasing number of iteration, but the large increase here will introduce delay time which make such system unsuitable for real time applications.

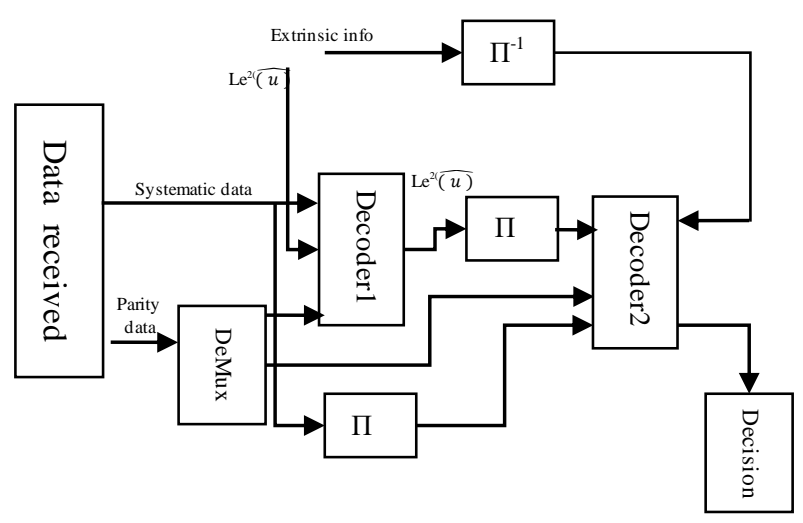

Figure 5. Basic Structure of an Iterative Turbo Decoder [32]

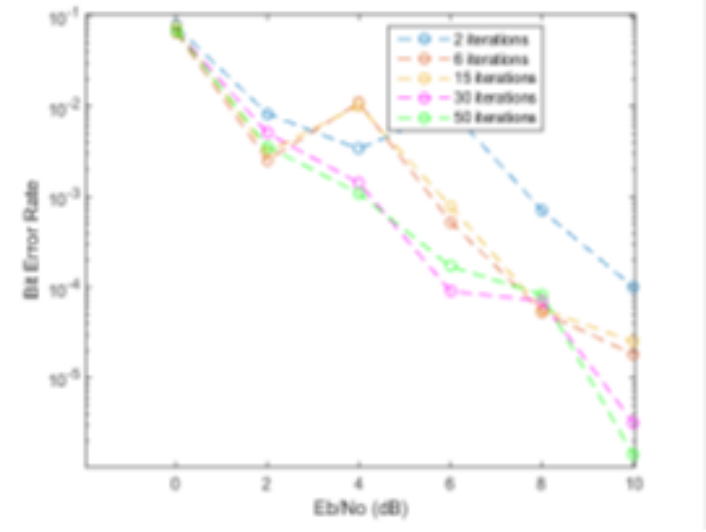

Figure 6. Performance of OSTBC-TURBO Codes for Various Iterations

\section{OVERVIEW ON BCH-TURBO-OSTBC}

Turbo codes dominate the research in error control coding together with low-density parity-check codes, due to their remarkable performances. Some research have been done to compare between LDPC and Turbo code in terms of performance, capacity and complexity [32], and It was concluded that the turbo code has better performance in moderate code rate.

Several concatenation schemes that combines OSTBC with turbo code have been proposed to improve bit error rate performance of an OSTBC receiver as we showed earlier [34].

Since It was verified In [10] and [35] that an outer BCH code have an effectiveness in improving the performance of a turbo code it is effective in lowering the error floor by correcting the residual errors, but it can also be used to alleviate other undesired effects of the iterative APP decoder. At the same time it improves the BER. We will study the concatenation of this scheme (BCH-TURBO) in concatenation with OSTBC in Rayleigh fading channel.

In this coding scheme, the outer encoder is BCH encoder and Inner Encoder is TURBO encoder. That is one block of data is first encoded by outer BCH encoder, and then encoded $\mathrm{BCH}$ code words are encoded by the inner TURBO encoder. After TURBO encoding the message packets of length $\mathrm{k}$, code words of length $\mathrm{K}$ are generated. These TURBO code words are OSTBC encoded using the matrix $\mathrm{G}$ and fed to the $\mathrm{N}$ transmitting antennas .as shown in Figure 7.The channels are assumed to be flat and the path gains from transmit antenna $n$ to receive antenna $m$ are defined as $\alpha_{n, m}$.

At the receiver side the OSTBC decoder use metrics as shown in Equations (4) and (5) to decode and detect symbols, these values are further processed at a TURBO decoder and BCH decoder successively.

The length of code word for $\mathrm{BCH}$ code is $\mathrm{n}=15$, while the message $\mathrm{k}=5$ only so the code rate is 0.33.And the TURBO code accept block length of 1500 with 8 -states rate $1 / 2$ convolutional component codes. 


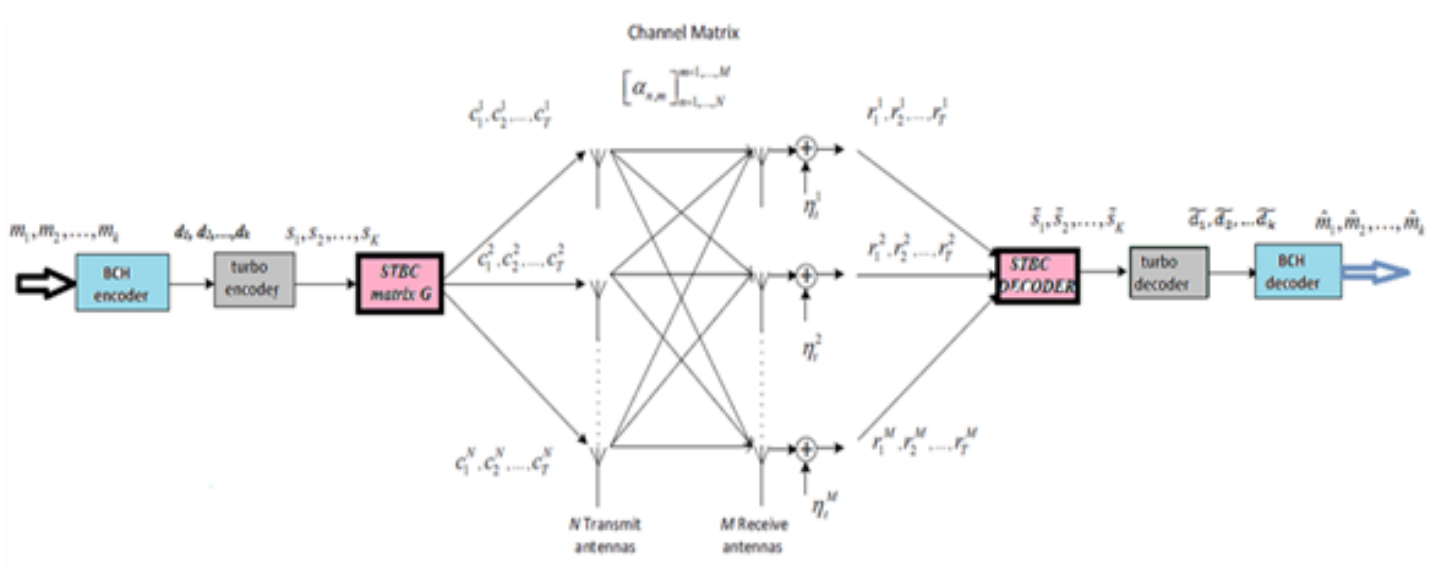

Figure 7. Proposed Model STBC Diagram with TURBO-BCH code

\section{RESULTS AND DISCUSSION}

In this section, simulations are performed to show the BER performance of following schemes, the results are evaluated for 2x1 MIMO system with 16-rectangular QAM modulation:

1. First the performance of different FEC in AWGN channel

2. OSTBC concatenated with different FEC (BCH, RS, CC, TCM, TURBO code) over Rayleigh fading channel.

3. Comparis on of OSTBC, OSTBC-Turbo and OSTBC- BCH-TURBO over Rayleigh fading channel. And finally the Performance of OSTBC-Turbo-BCH with and without two interleavers.

Parameters of Different Channel Encoders Used in simulations are summarized in Table 1.

Table 1. Parameters for Various Codes

\begin{tabular}{|c|c|c|}
\hline Code & $\begin{array}{c}\text { Encoder } \\
\text { parameters }\end{array}$ & $\begin{array}{c}\text { Decoder } \\
\text { parameters }\end{array}$ \\
\hline TURBO & $\begin{array}{l}8 \text { state, rate } 1 / 2 \\
\text { convolutional } \\
\text { component codes }\end{array}$ & $\begin{array}{l}\text { APP algorithm } \\
\text { With } 4 \text { iterations }\end{array}$ \\
\hline $\mathrm{CC}$ & $\begin{array}{l}8 \text { states } \\
\text { Rate } 1 / 2\end{array}$ & Viterbi algorithm \\
\hline $\mathrm{BCH}$ & $\begin{array}{l}\text { codeword } \\
\text { length=15/ } \\
\text { Message } \\
\text { length=5 }\end{array}$ & $\begin{array}{l}\text { Berlekamp } \\
\text { Algorithm }\end{array}$ \\
\hline $\begin{array}{c}\text { REED } \\
\text { SOLOMON }\end{array}$ & $\begin{array}{c}\text { codeword } \\
\text { length=7/ } \\
\text { Message } \\
\text { length=3 }\end{array}$ & $\begin{array}{c}\text { Berlekamp- } \\
\text { Massey decoding }\end{array}$ \\
\hline TCM & $\begin{array}{l}16 \text { rectangular } \\
\text { QAM } \\
4 \text { state trellis }\end{array}$ & Viterbi algorithm \\
\hline
\end{tabular}

a. Performance Of Different FEC In AWGN Channel

Figure 8 shows the performance of different FEC in AWGN channel, the TURBO code and TCM are very efficient in AWGN. They provide more than $4 \mathrm{~dB}$ coding gain at $10^{-6}$ over others codes.

b. OSTBC concatenated with different FEC (BCH, RS, CC, TCM, TURBO code).

Performance of OSTBC is improved due to the use of a powerful FEC. Results for TURBO or TCM show better performance than OSTBC only or OSTBC with $\mathrm{CC}, \mathrm{BCH}$, and RS. TURBO-OSTBC provides $12 \mathrm{~dB}$ at BER $\sim 10^{-5}$ over uncoded OSTBC and $8 \mathrm{~dB}$ over OSTBC-TCM, as show in Figure 9. 


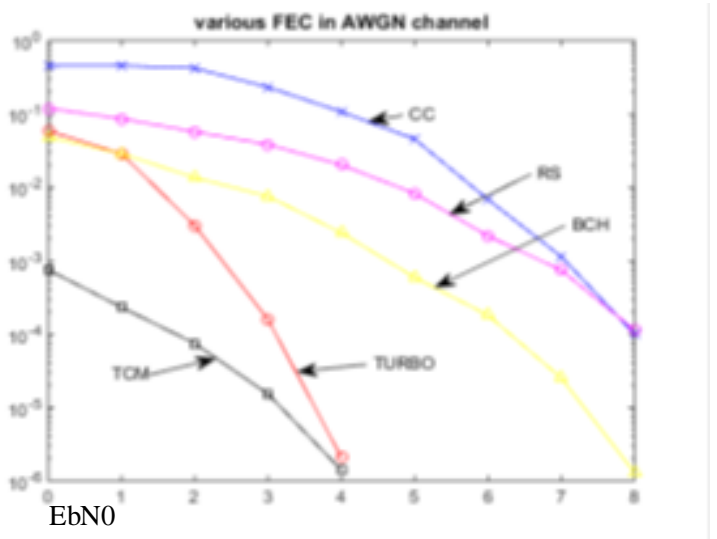

Figure 8. Simulation of Different FEC without OSTBC in AWGN Channel

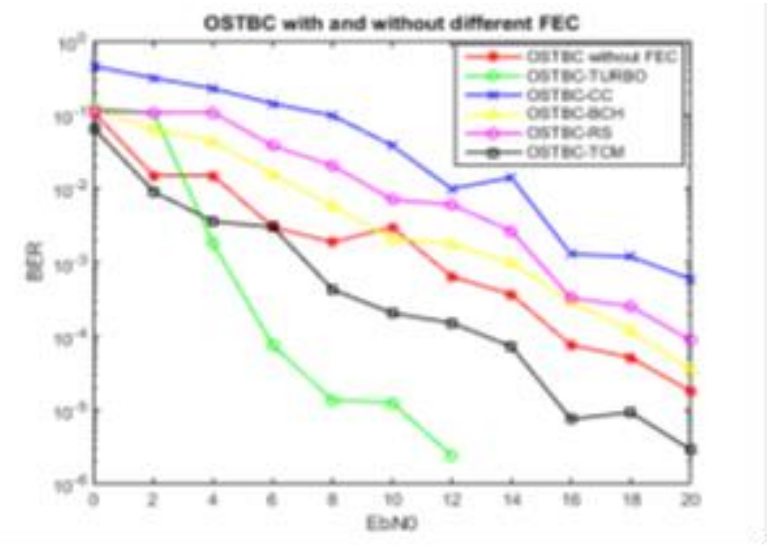

Figure 9. Concatenated OSTBC with Different FEC in Rayleigh Fading Channel

c. Comparison between OSTBC, OSTBC-Turbo and OSTBC- BCH-TURBO

Figure 10 shows that we can obtain an improvement of $5 \mathrm{~dB}$ at $10^{-6}$ when using BCH-TURBO in comparison with TURBO only. And in comparison with other concatenation of STBC and other coding schemes we can see that in [36] the BER is equal to $10^{-4}$ for $17 \mathrm{~dB}$ for $2 * 1$ MIMO channel. Otherwise in our system we can obtain BER of $10^{-4}$ for EbN0 of $5 \mathrm{~dB}$. In other contribution [6] they can obtain $9 \mathrm{~dB}$ improvement at $2 * 10^{-4}$ BER with LDPC concatenated with STBC.

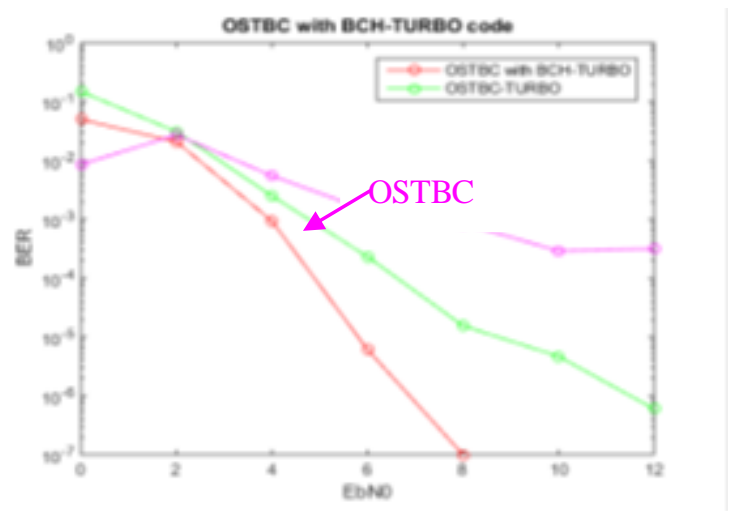

Figure 10. Performance Comparis on of OSTBC with BCH-TURBO or without it 
d. Performance of OSTBC-Turbo-BCH with and without different interleavers.

Figure 11 shows the performance of $\mathrm{BCH}$-TURBO system with and without interleaver, the $\mathrm{BCH}-$ TURBO with block interleaver and matrix interleaver provide just $1 \mathrm{~dB}$ at $10^{-6}$ at the cost of introducing additional time-delay, memory space requirements, and system complexity. So we can relinquish its use in our system.

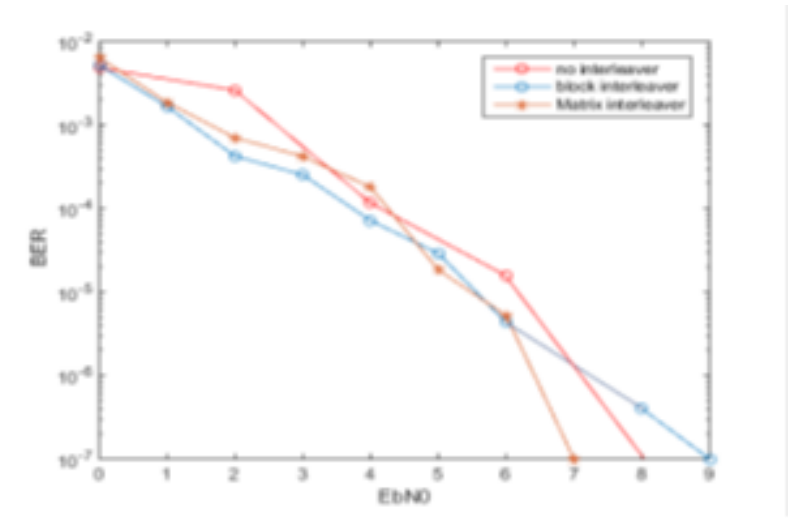

Figure 11. BCH-TURBO with OSTBC with Different Interleaver

\section{CONCLUSION}

In this paper, we have tried to give a clear outline of the effect of using a serially concatenated BCH-TURBO code with OSTBC in multiple antennas on BER performance of wireless communication systems.

The effectiveness of an outer $\mathrm{BCH}$ code in improving the performance of a turbo code was verified by simulation. According to the simulated results obtained for $\mathrm{BCH}$ only and TURBO only codes, we observe as a conclusion that for $\mathrm{BCH}$ coding system to achieve BER of $10^{-5}$ the $\mathrm{E}_{\mathrm{b}} \mathrm{N}_{0}$ should be maximum, it means that the signal strength should be very high.

When we observe TURBO codes to achieve BER of $10^{-6}$ the Eb/N0 should be over $10 \mathrm{db}$ with 50 iterations, for this, to achieve better BER we need to increase the number of iterations at the decoding stage as we see from the Figure 6. Increasing of the number of iterations will increase execution time. Thus, to remedy this disadvantage we need to propose a coding scheme which can show zero BER under low $\mathrm{E}_{\mathrm{b}} / \mathrm{N}_{0}$. Concatenation of $\mathrm{BCH}$ and TURBO in OSTBC system is proposed and the results $\left(10^{-6}\right.$ at $6 \mathrm{~dB}$ with only 4 iterations) of this coding scheme prove that such a framework is effective channel code to achieve low BER that is appropriate for a high quality wireless communications, because, the overall system provides, with simple addition to the standard turbo in OSTBC system, a significant improvement in BER performance with a small increasing in complexity of decoding.

In the next step, we will simulate our system with more than 2 antennas and we will realize the software and physical prototyping of our system.

\section{REFERENCES}

[1] E.Teletar, "Capacity Of Multi Antenna Gaussian Channels," emerging telecommunications technologies , vol. 10, pp. 585-595, Nov,Dec 1999.

[2] G.J. Foschini And M.Gans, "On Limits Of Wireless Communications In Fading Environment When Using Multiple Antennas," Wireless Pers. Commun.Kluwer Academic Publishers, vol. 6, pp. 311-335, 1998.

[3] S.M. Alamouti, "A Simple Transmit Diversity Technique For Wireless Coomunications," IEEE Journal On Selected Areas In Comm, vol. 16, pp. 1451-1458, Oct.1998.

[4] V.Tarokh, "Space-Time Block Codes From Orthogonal Designs," IEEE Trans On Information Theory, vol. 45, no. 5, pp. 1456-1467, July 1999.

[5] T. H. Liew, J. Pliquett, B. L. Yeap, and L. Yang, "Concatenated Space Time Block Codes And TCM, Turbo TCM, Convolutional As Well As Turbo Codes," ,Global Telecommunications Conference,IEEE GLOBECOM 2000, vol. 3, pp. 1829-1833, 2000.

[6] Elies Ghay oula, Mohamed Haj Taieb, Jean-Yves Chouinard, Ridha Ghayoula, and Ammar Bouallegue, "Improving MIMO System Performances By Concatenated LDPC Decoder To The MRC And STBC Receivers," World Symposium On Computer Networks And Information Security, 2015.

Indonesian J Elec Eng \& Comp Sci, Vol. 11, No. 3, September 2018 : 898 - 907 
[7] Ilesanmi Banjo Oluwafemi, "HybridConcatenated Coding Scheme for MIMO Systems," international journal of electrcal and computer engineering (IJECE), vol. 5, no. 3, pp. 464-476, june 2015.

[8] Isiaka Ajewale Alimi, Kayode Francis Akingbad, Jide Julius Popoolae, and Michael O. Kolawole, "An Hybrid Coding Technique for Efficient Bandwidth Usage in conformity with IEEE 802.11 WLAN Standard," international journal of electrical an computer engineering (IJECE), vol. 3, no. 5, pp. 593-602, october 2013.

[9] C. Berrou, A. Glavieux, and P. Thitimajshima, "Near Shannon Limit Error-Correcting Coding And Decoding: Turbo Codes," In Proc. 1993 IEEE Int. Communications Conference, pp. 1064-1070, May 1993.

[10] Oscar Y. Takeshita, Oliver M. Collins, Peter C. Massey, and Daniel J. Costello, "On The Frame Error Rate Of Concatenated Turbo Codes," IEEE Transactions On Communications, vol. 49, no. 4, APRIL 2001.

[11] V Tarokh, H. Jafarkhani, and A. R. Calderbank, "Space Time Block Coding For Wireless Communications : Performance And Results," IEEE Journal On Selected Areas In Comm, vol. 17, no. 3, pp. 451-560, Mar 1999.

[12] G.D Forney, "Concatenated Codes ," Massachusetts Institute Of Technology Research Laboratory Of Electronics, Technical Report 440, december I, 1965.

[13] P Sweeny, Error Control Coding, From Theory To Practice ,pp35. University of Surrey, Guildford, UK: WILEY, 2002.

[14] C. Langton, "Coding And Decoding With Convolutional Code," July 1999

[15] A Viterbi, "Error Bounds For Convolutional Codes And An Asymptotically Optimum Decoding Algorithm," IEEE Trans, Inform,Theory, vol. IT-13, pp. 260-269, Apr 1967.

[16] L.R.Bahl, J. Cocke, F. Jelinek, and J. Raviv, "Optimal Decoding Of Linear Codes For Minimizing Symbol Error Rate," IEEE, Trans , ,Inform,Theory, pp. 284-287, Mar 1974.

[17] Y.J. Ko and Jung-Im Kim, "Serial Concatenation Of Space-Time And Recursive Convolutional Codes," ETRI Journal, vol. 25, April 2003.

[18] Lucian Andrei Perişoară, "BER Analysis Of STBC Codes For MIMO Rayleigh Flat Fading Channels," Telfor Journa, vol. 4, no. 2, 2012.

[19] S. Hyun-Jun, Kang. Chul-Gyu, and Chang-Heon Oh, "Performance Analy sis Of STBC System Combined With Convolution Code Fot Improvement Of Transmission Reliability," The Journal of Advanced Navigation Technology, vol. 15, 2011.

[20] Ray C. Bose and Dwijendra K. Ray-Chaudhuri, "On A Class Of Error Correcting Binary Group Codes. Information And Control," Information and Control, vol. 3, pp. 68-79, 1960.

[21] Alexis Hocquenghem, "Codes Correcteurs D’erreurs ," Chiffres, vol. 2, pp. 147-156, 1959.

[22] D.Costello and S.Lin, Error Control Coding Fundamentals And Applications.: printice hall, 2004.

[23] Girish Kumar and M N Sree Ranga Raju, "Design And Performance Evaluation Of Error Detection And Correction Using Concatenated BCH And LDPC Coding Scheme For Data Streams In Satellite Communication," International Journal Of Engineering ResearchResearch And Technology, vol. 4, August 2015.

[24] P.A Martin and D.P.Taylor, "High-Throughput Error Correcting Space-Time Block Codes," International Symposium On Information Theory, ISIT 2004.

[25] Roopali Agarwal and Manoj K. Shukla, "SC-FDM-IDM A Scheme Employing BCH Coding," International Journal Of Electrical and Computer Engineering (IJECE), vol. 7, no. 2, pp. 992-998, 2017.

[26] Samridhi and Jyoteesh Malhotra, "Investigation Channel Coding Technique," International Journal Of Computer Applications, vol. 115, no. 3, April 2015.

[27] M. Lalame, Karine Amis, and D Leroux, "On The Use OfReed-Solomon Codes In Space-Time Coding," IEEE 2005 , 16th International Symposium On Personal , And Mobile Radio Communications, vol. 4, July 2006.

[28] Abderrahmane Anou, Mustapha Djebari, Merouane Mehdi, and Messaoud Bensebti, "Diversity Techniques To Combat Fading In Wimax," 7th WSEAS Int. Conf. On Electronics, Hardware, Wireless And Optical Communications, Cambridge, UK, February 20-22, 2008

[29] P. Srinivasa Rao et al., "Performance Analysis Of MIMO Systems Using TCM And Comparison With OSTBC," International Journal Of Future Generation Communication And Networking, vol. 6, aug 2013.

[30] Mehdi Teimouri and Mohsen Shiva, "Concatenated Space Time Block Coding With Trellis Coded Modulation Over Fading Channels In The Absence Of Channel State Information," IEEE Transaction In Wireless Communications, vol. 7, no. 12 , December 2008.

[31] B.Revathi, P.S.Samhitha, V.V.Lavania:G.Amani, and N.A.Manikantha, "Ber Analysis Of Tcm Aided Stbc In Mimo-Ofdm Systems," International Conference On Electrical, Electronics, Signals, Commmunication Ans Optimization, IEEE 2015, September 2015.

[32] Alaa Eldin.S.hassan, Moawad Dessouky, Atef Abou Elazm, and Mona Shokair, "Evaluation Of Complexity Versus Performance For Turbo Code And LDPC Under Different Code Rates," SPACOMM 2012.

[33] J.P.K. Chu and P.J. Mclane, "Serial Concatenation Of Stbc Or Dstbc With Convolutional Codes Or Turbo Codes For Space-Time Correlated Channels," Wireless Communication And Networking Conference, IEEE 2004, vol. 4, 2004.

[34] Junghoon Suh and M. M. K. Howlader, "Concatenated Of Turbo Code With Transmit Diversity," VTC Spring 2002. IEEE 55th, vol. 3, pp. 1228 - 1232, 2002.

[35] J. D. Andersen, "Turbo Codes Extended With Outer Bch Code," Turbo Codes Extended With Outer Bch Code," Electronics Letters, vol. 32, pp. 259-260, Oct 1996.

[36] Guangxi Zhu, Yejun He, Gan Liu, Bijun Zhang, and Feng Wang, "Concatenation Of Space-Time Block Codes And Turbo Product Codes Over Rayleigh Flat Fading Channels," VTC Spring2005, IEEE 2005. 